\title{
Respuesta frente al Covid-19 para la atención de la población migrante mexicana en el sur de Estados Unidos
}

\section{Response to Covid-19 for the care of the Mexican migrant population in the southern United States}

\author{
Ana María López-Jaramillo, María Gudelia Rangel-Gómez \\ y Rodolfo Cruz-Piñeiro
}

El Colegio de la Frontera Norte, México

\section{Resumen}

Una de las interrogantes actuales es sobre el impacto de la pandemia causada por la Covid-19, SARS-CoV-2 sobre los grupos de personas de mayor vulnerabilidad. En general se ha documentado que la pandemia está afectando de manera desigual a diferentes poblaciones. Por ello, este trabajo exploratorio indaga sobre el impacto que está teniendo el Covid-19 sobre la población de inmigrantes mexicanos que residen en Estados Unidos. Para ello, se considera una muestra de migrantes mexicanos que han sido atendidos a través del Programa de Unidades Móviles de Salud y Bienestar de la Comisión de Salud Fronteriza México-Estados Unidos en las ciudades de Phoenix y Tucson, Arizona. El trabajo también explora y analiza las primeras acciones realizadas en la región fronteriza por los actores gubernamentales de ambos países. Entre los resultados se destaca que más de la mitad de la población reportó tener diabetes mellitus, una alimentación moderada o alta en grasas y azúcares y poca actividad física; así también se observó que son una población con una estancia promedio en Estados Unidos de alrededor de 12 años, con un nivel menor a la preparatoria y bajo nivel de inglés, y labora en ocupaciones de baja remuneración salarial.

Palabras clave: Covid-19, Inmigrantes mexicanos, Estados Unidos, Unidades móviles de salud y bienestar.

\section{Abstract}

One of the current questions is about the impact of the pandemic caused by Covid-19, SARS$\mathrm{CoV}-2$ on the most vulnerable groups of people. In general, it has been documented that the pandemic is affecting different populations unequally. Therefore, this exploratory work investigates the impact that Covid-19 is having on the population of Mexican immigrants residing in United States. To do this, a sample of Mexican migrants who have been treated through the Program of Mobile Health and Welfare Units of the United States-Mexico Border Health Commission in the cities of Phoenix and Tucson, Arizona, is considered. The work also explores and analyzes the first actions carried out in the border region by government actors from both countries. Among the results, it stands out that more than half of the population reported having diabitis mellitus, a moderate or high fat and sugar diet and little physical activity; Thus, it was also observed that they are a population with an average stay in the United States of around 12 years, with a level lower than high school and a low level of English, and work in low-wage occupations.

Key words: Covid-19, Mexican immigrants, United States, mobile health and wellness units. 


\section{INTRODUCCIÓN}

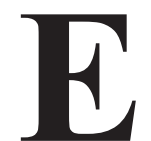

1 sistema migratorio de la región Centro y Norteamérica se ha caracterizado por mostrar procesos de movilidad muy diversos. Los flujos migratorios entre los países de esta región, Honduras, El Salvador, Guatemala, México y Estados Unidos han cambiado sustancialmente en los últimos años. La histórica migración de mexicanos a Estados Unidos ha disminuido a números minúsculos comparados con los de la década de los noventa. Sin embargo, los movimientos de personas de los países centroamericanos hacia el norte se han incrementado sustancialmente. Así también los movimientos de migrantes realizando los retornos a sus países de origen se ha incrementado, al igual que el número de deportaciones y el volumen de los migrantes en tránsito por territorio mexicano. Todo ello ha confluido en generar un sistema migratorio más complejo y diverso en esta región del planeta. La movilidad entre estos países ha estado conformada principalmente por poblaciones vulnerables, poblaciones que han tenido que buscar otras opciones de vida y bienestar en otras latitudes. En gran medida, en los últimos años hemos sido testigos que estos flujos migratorios se han conformado por familias de migrantes, mujeres y niños, algo diferente de hace un par de décadas, que estaban compuestas principalmente por jóvenes hombres en edad productiva. Todo ello muestra un panorama bastante difícil de atención para esta población en movimiento.

A inicios del año 2020, particularmente desde marzo, esta región se vió fuertemente impactada por la pandemia mas cruenta de los últimas décadas o siglos, el SARS-CoV-2, Covid-19, que ha cobrado un gran número de vidas en esta región. Hasta octubre de 2020, en México han fallecido más de 85 mil personas y el nivel de contagio se ha ido incrementando en las últimas semanas. La pandemia es una gran amenaza para las poblaciones en general, pero para aquellas poblaciones en situación de vulnerabilidad los riesgos se incrementan y su impacto podría ser aun más grave. Las desigualdades se incrementan con el impacto de la pandemia, en aquellas poblaciones marginadas y que cuenten con menores posibilidades. Estados Unidos es uno de los países que ha sido más afectado por el Covid-19. En este país residen alrededor de 11.7 millones de mexicanos, alrededor de 4.9 millones de mexicanos sin documento. Asimismo, la Oficina de Salud de la Minorías en Estados Unidos ha señalado que el índice más alto de falta de cobertura médica lo tienen los latinos, la mayoría integrada por migrantes mexicanos especialmente aquellos migrantes que no cuentan con 
documentos de residencia en ese país. Esta situación hace a la población migrante mexicana más vulnerable ante otro grupo étnico que reside en los Estados Unidos. El acceso y utilización de los servicios de salud por parte de personas indocumentadas varía según la entidad y ciudad de residencia, pero existen elementos comunes que determinan la situación de vulnerabilidad que experimenta este grupo de migrantes connacionales.

Por otro lado, en las regiones o zonas de mayor movilidad poblacional suele ser de mayor complejidad el control del virus, la movilidad incrementa el riesgo de contagio, en zonas fronterizas, lo cual ha sido un gran reto. Una de las preguntas que nos podemos hacer es cómo ha estado impactando esta pandemia en aquellas zonas fronterizas, particularmente en las ciudades que se ubican en la región fronteriza entre México y Estados Unidos; además preguntarnos qué tipo de medidas y mecanismos se han estado implementando por los sistemas de salud de ambos lados de la frontera o del lado mexicano, para atenuar los efectos del impacto de esta pandemia. Pero también nos podríamos preguntar cómo ha estado impactando a la población de los migrantes mexicanos que residen en Estados Unidos.

Considerando este contexto, el presente trabajo tiene como objetivo general explorar el impacto que esta teniendo el Covid-19 sobre la población migrante mexicana que radica en Estados Unidos, además de explorar y analizar las primeras medidas que se han implementado por parte de los gobiernos federales y estatales en los estados fronterizos de ambos países. En la primera sección abordaremos las acciones efectuadas por los actores gubernamentales de México y Estados Unidos; en la segunda, se hará un análisis exploratorio de los impactos de la pandemia sobre la población mexicana en Estados Unidos, a través del Programa de Unidades Móviles de Salud y Bienestar de la Sección mexicana de la Comisión de Salud Fronteriza México-Estados Unidos, como parte del fortalecimiento estratégico de la Ventanilla de Salud. Este análisis se hará considerando una muestra de migrantes mexicanos que han sido atendidos por este servicio de salud, en las ciudades de Phoenix y Tucson, Estados Unidos. Finalmente, se presentan los retos y recomendaciones para seguir atendiendo esta pandemia entre los migrantes mexicanos que viven en los estados fronterizos entre México y Estados Unidos.

\section{Primeras respuestas de los actores gubernamentales EN los ESTados fronterizos MÉXICO - Estados Unidos}

Entre las acciones y buenas prácticas que se han llevado a cabo en materia de Covid-19, en la región fronteriza México-Estados Unidos, destaca 
el fortalecimiento de la capacidad técnica del personal de salud y otros involucrados, acciones específicas a distancia de apoyo a la salud mental durante la pandemia, el desarrollo de material educativo de prevención, la implementación de filtros ambulatorios, así como de clínicas y hospitales temporales, la ejecución de clínicas de fiebre en el primer nivel de atención, además de la realización de pruebas con unidades móviles.

Destaca la vinculación y articulación interinstitucional en la región noreste (Nuevo León, Coahuila y Tamaulipas), para el monitoreo y atención médica a la población migrante en estaciones migratorias, repatriación y albergues, en colaboración con el Instituto Nacional de Migración, además de la coordinación para fortalecer acciones en conjunto. Asimismo, Texas implementó una herramienta de consulta estatal para dar seguimiento al número de casos, pruebas y defunciones por sexo, raza y grupo de edad, con especial énfasis en la frontera con México.

Entre las buenas prácticas binacionales, destaca la activación del Protocolo Operacional para la Comunicación y Coordinación Binacional entre México y Estados Unidos sobre Notificaciones de Enfermedades y Brotes, además de la coordinación y comunicación entre Sonora-Arizona, así como Baja California-California, mediante el intercambio de información diaria y el manejo de casos binacionales.

De manera general, Estados Unidos ha incrementado la disponibilidad y frecuencia de las pruebas de Covid-19 a la población, a través de distintas colaboraciones entre la academia y agencias públicas y privadas. Entre las innovaciones, destaca el desarrollo de un app de salud digital para teleconsulta en Chihuahua; así como el monitoreo de pacientes positivos por videollamada en Nuevo León.

\section{Situación del Covid-19 en los ESTAdos Fronterizos México-Estados Unidos}

Según los reportes oficiales de la Secretaría de Salud, al 27 de octubre de 2020, los estados fronterizos del norte de México reportan un acumulado de más de 193 mil casos positivos de Covid-19, y más de 17 mil defunciones (ver Tabla 1).

De acuerdo con información de Centros para el Control y la Prevención de Enfermedades (CDC), al 27 de octubre de 2020, en los estados fronterizos de Estados Unidos con México, se reportan más de 2 millones de casos y más de 42 mil defunciones por el Covid-19 (ver Tabla 2). 
Respuesta frente al Covid-19 para la atención de la población migrante mexicana ... / A.M. LÓPEZ JARAMILLO et al.

Tabla 1: Reporte de casos en estados fronterizos México-Estados Unidos México, marzo-octubre de 2020

\begin{tabular}{lrrrr}
\hline Estado & $\begin{array}{r}\text { Casos } \\
\text { positivos }\end{array}$ & $\begin{array}{r}\text { Casos } \\
\text { negativos }\end{array}$ & $\begin{array}{r}\text { Casos } \\
\text { sospechosos }\end{array}$ & Defunciones \\
\hline Baja California & 22,631 & 15,583 & 23,485 & 3,842 \\
Sonora & 37,698 & 25,410 & 5,167 & 3,103 \\
Chihuahua & 16,928 & 13,608 & 10,513 & 1,816 \\
Coahuila & 32,490 & 42,270 & 9,922 & 2,284 \\
Nuevo León & 51,785 & 58,087 & 15,709 & 3,770 \\
Tamaulipas & 31,903 & 40,906 & 7,590 & 2,580 \\
Total & 193,435 & 195,864 & 72,386 & 17,395 \\
\hline
\end{tabular}

Fuente: DGE, 27 de octubre de 2020, https://datos.covid-19.conacyt.mx/

Tabla 2: Reporte de casos en estados fronterizos México-Estados Unidos, Estados Unidos, marzo-octubre de 2020

\begin{tabular}{lrr}
\hline Estado & Casos & Defunciones \\
\hline California & 908,713 & 17,475 \\
Arizona & 241,165 & 5,905 \\
Nuevo Mexico & 43,826 & 991 \\
Texas & 879,994 & 17,700 \\
Total & $2,073,698$ & 42,071 \\
\hline
\end{tabular}

Fuente: CDC, 27 de octubre de 2020, https://covid.cdc.gov/covid-data-tracker/index.html\#cases_casesper $100 \mathrm{k}$

Por otra parte, la Secretaría de Relaciones Exteriores en su Nota informativa No. 36, en la que presenta la actualización sobre el seguimiento de connacionales con Covid-19 en Estados Unidos, se destaca que al 31 de agosto de 2020 fallecieron, 2,270 connacionales.

Asimismo, al revisar las proporciones en los estados de la frontera con México, se destaca que sólo los cuatro estados fronterizos concentran más de una tercera parte (33.5 por ciento) de las defunciones por Covid-19 de la población mexicana en Estados Unidos (Mapa 1). 
Mapa 1: Mexicanos fallecidos por Covid-19 en Estados Unidos, marzo a agosto de 2020

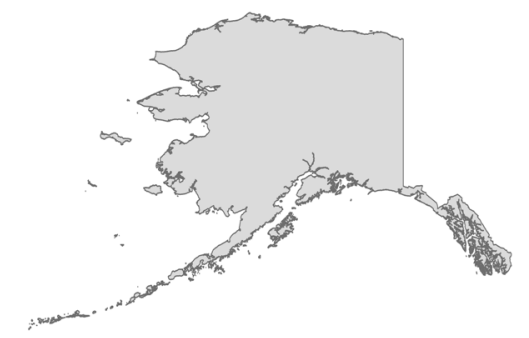

\section{Cuartiles}
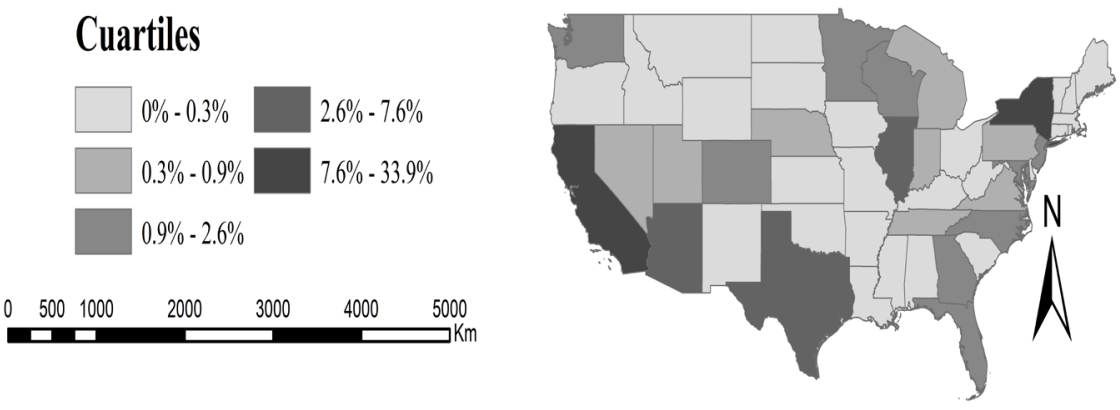

* Incluye Connecticut y condados de Nueva Jersey.

Las cifras corresponden a la información reportada a los consulados y podrían variar.

Fuente: elaboración propia a partir de datos de la Secretaría de Relaciones Exteriores, 31 de agosto de 2020. Disponible en https://www.gob.mx/sre/documentos/nota-informativa-relaciones-exteriores-no-36.

\section{El CASO DE LA POBLACIÓN MIGRANTE MEXICANA ATENDIDA EN LAS Unidades Móviles de Salud y Bienestar (UMSB)- Mobile Health and Wellness (MHW) Units en Estados Unidos}

El Programa de Unidades Móviles de Salud y Bienestar (UMSB), de la Sección mexicana de la Comisión de Salud Fronteriza México-Estados Unidos (CSFMEU), como parte del fortalecimiento de la Estrategia Ventanillas de Salud (VDS), provee servicios ambulatorios, información y capacitación para la prevención de enfermedades y la promoción de la salud entre la población latina residente en comunidades alejadas y de difícil acceso a servicios de salud, en áreas metropolitanas de Chicago (Illinois), Dallas (Texas), Los Ángeles (California), Phoenix (Arizona) y Nueva York en su primera fase. Posteriormente, se implementaron las UMSB en Denver (Colorado), Las Vegas (Nevada), Tucson (Arizona), Miami y Orlando (Florida), y Raleigh (Carolina del Norte). Su principal objetivo es brindar 
orientación en salud sobre temas prioritarios como nutrición, obesidad, diabetes, salud de la mujer, salud infantil, salud mental, VIH/SIDA, adicciones. Así mismo, ofrece información sobre violencia doméstica, acceso a servicios de salud y educativos, orientación legal y financiera.

Las UMSB difunden, mensualmente, la ubicación y los horarios en que estarán brindando los servicios. Esta difusión la hacen por distintos medios, de acuerdo a su ubicación y alianzas. De esta manera, algunas UMSB utilizan redes sociales, medios de comunicación local y volanteo, principalmente.

En cuanto a la logística de atención, cuando es presencial, depende de la cantidad de personas que asistan, pudiendo brindar la atención personalizada o en grupos. Actualmente ante la pandemia por el Covid-19, la atención se brinda personalizada vía telefónica, o en grupos a través de redes sociales.

Las UMSB cuentan con un sistema de registro, en el cual está contenida la base de datos con información demográfica y epidemiológica de las personas que recibieron algún tipo de servicio. Para registrar esta información se cuenta con el consentimiento informado de cada uno de los usuarios quienes verbalmente autorizaron dar información.

A partir de la contigencia de salud a nivel mundial, los retos que ésta implica, y teniendo en cuenta la importancia de conocer y mejorar la salud de la población migrante mexicana en Estados Unidos, se llevó a cabo la adaptación de los servicios de las UMSB, en materia de Covid-19, la cual incluye orientación, detección y referencia. Destaca el especial énfasis a la atención a la salud mental, por lo que, en coordinación con la Facultad de Psicología de la Universidad Nacional Autónoma de México, se está trabajando en la implementación del Tamizaje de Salud Mental, para identificar diversos padecimientos y hacer referencias oportunas. Asimismo, la Sección mexicana de la CSFMEU, la Dirección General de Relaciones Internacionales de la Secretaría de Salud de México y la Universidad de Arizona, desarrollaron un cuestionario relacionado con el Covid-19, para conocer la situación de la población migrante mexicana en Estados Unidos, frente a la pandemia por el Covid-19.

La prueba piloto del Cuestionario de Covid-19, dio inicio en el mes de marzo, en las UMSB de Tucson y Phoenix, Arizona, y posteriormente, a partir de mediados del mes de abril se integró para la aplicación de todas las UMSB. A continuación, se presentan los resultados sociodemográficos de las personas que acudieron a las UMSB y que recibieron dicha información entre marzo y agosto de 2020. El principal objetivo será describir 
la respuesta y los retos binacionales frente al Covid-19 y presentar el perfil demográfico de la población migrante en Estados Unidos, que recibió información sobre Covid-19 entre ese periodo en las UMSB.

\section{Material y MÉtodo}

\section{Fuente de información}

Las UMSB utilizan un sistema de base de datos que captura información sobre las personas atendidas y los servicios prestados. Éstas se ubicaron estratégicamente en ciudades objetivo en función de la concentración de población hispana determinada por la red de oficinas consulares en los Estados Unidos y la Sección de México de la CSFMEU, así mismo, se consideraron como prioritarias aquellas comunidades alejadas y de difícil acceso a servicios de salud.

\section{Análisis descriptivo}

Los análisis descriptivos se derivan de fuentes de datos secundarias sin identificadores a nivel individual. Las características demográficas y epidemiológicas de la población objetivo se resumieron desde un nivel ecológico (es decir, sólo se dispone de datos resumidos de la población). Se calcularon estimaciones de prevalencia para estados patológicos comúnmente identificados y datos de recuento para exámenes de detección de salud, educación para la salud y servicios de derivación proporcionados a través de las UMSB. Como este análisis utilizó datos secundarios y no se consideró investigación con sujetos humanos, no se requirió aprobación ética.

Es necesario precisar que los datos presentados corresponden a información proporcionada por usuarios que se acercaron a las 2 UMSB, con las que inició la prueba piloto del Cuestionario de Covid-19, entre marzo y agosto de 2020. El volumen de información corresponde a 73 por ciento de la UMSB de Tucson y 27 por ciento a Phoenix.

\section{Resultados}

A partir de la contigencia de salud a nivel mundial y teniendo en cuenta la importancia de conocer y mejorar la salud de la población migrante mexicana en Estados Unidos, las UMSB implementaron un módulo de información relacionado con el Covid-19. A continuación se presentan los resultados sociodemográficas de las personas que acudieron a dichas unidades y recibieron información entre marzo y agosto de 2020. 
Respuesta frente al Covid-19 para la atención de la población migrante mexicana ... / A.M. LÓPEZ JARAMILLO et al.

\section{Perfil sociodemográfico de los inmigrantes mexicanos en Estados Unidos}

Lugar de origen

Entre marzo y agosto de 2020, en las UMSB se proporcionó información sobre Covid-19 a 891 migrantes mexicanos que viven en Estados Unidos. Aproximadamente 45 por ciento de las personas son originarias del estado de Sonora, dado que como se mencionó anteriormente, la mayoría de la población se acercó a las UMSB de Tucson y Phoenix, las cuales respondieron a la prueba piloto del formulario de Covid-19. Por otra parte, los estados de la región tradicional de la migración mexicana (Jalisco, Michoacán, Guanajuato, Zacatecas y Sinaloa), aportaron la cuarta parte de las personas (Mapa 2).

Mapa 2: Estado de origen de personas atendidas en Unidades Móviles de Salud y Bienestar, marzo a agosto de 2020

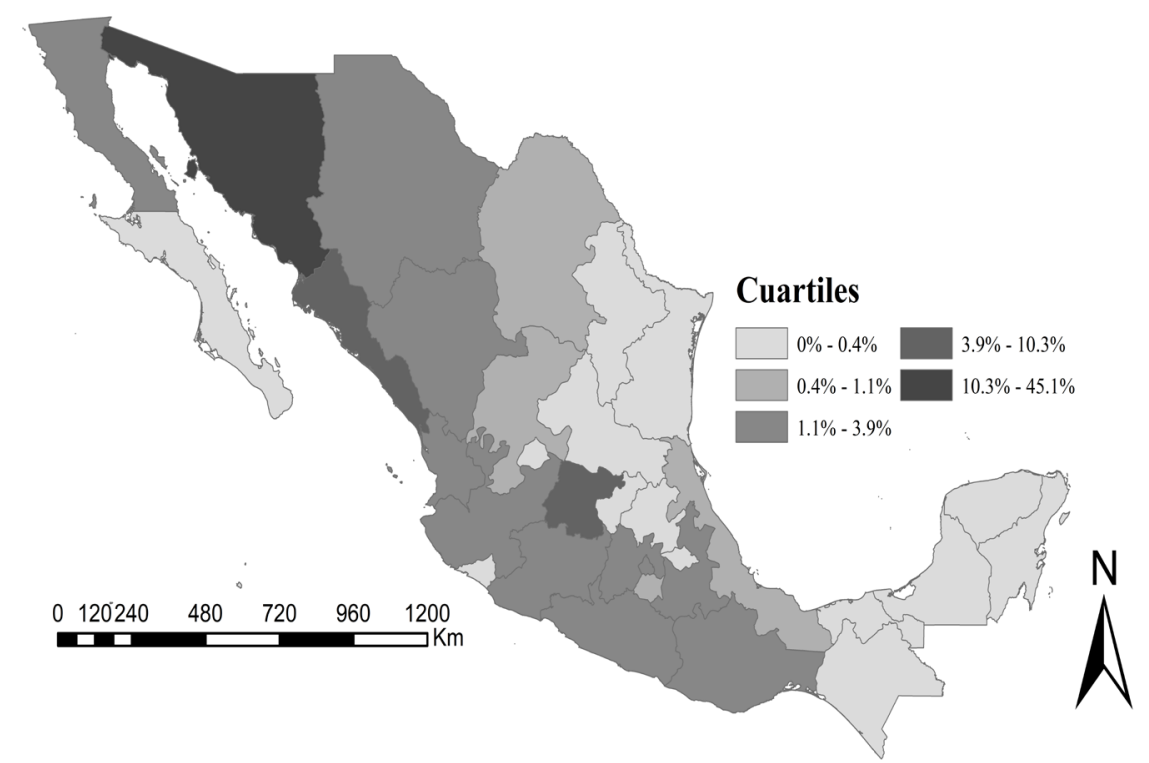

Fuente: elaboración propia a partir de información en Base de Datos UMSB 2020.

Estructura por edad y género

En cuanto a la estructura por edad y género de los migrantes atendidos en las diferentes UMSB y que recibieron información sobre Covid-19 entre marzo y agosto de 2020 se observó que la mayoría de la población corres- 
pondía a la femenina ( 68 por ciento) y según la edad, la mayor proporción (44 por ciento) se concentró en la población económicamente activa, es decir, 25 y 49 años (Figura 1).

Figura 1: Estructura por edad y sexo de la población informada. Unidades Móviles de Salud y Bienestar, marzo a agosto de 2020

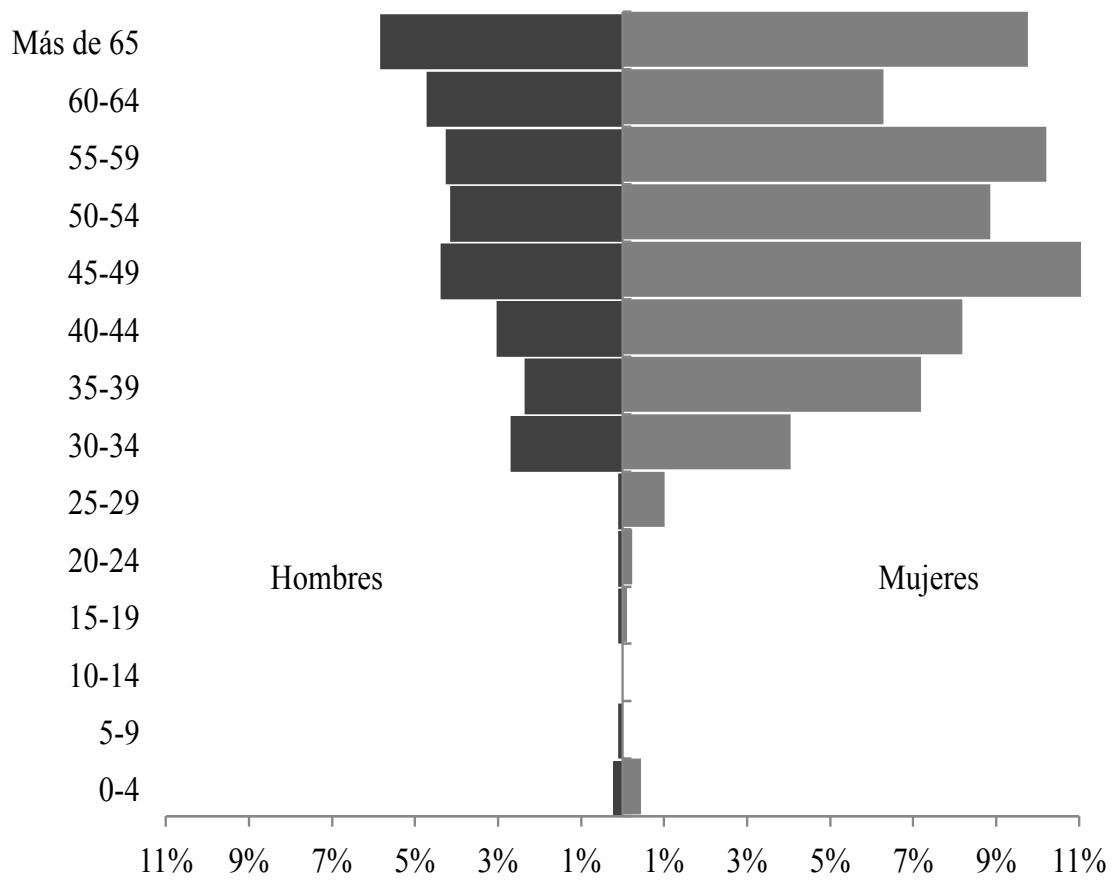

Fuente: elaboración propia a partir de información en Base de Datos UMSB 2020.

Años viviendo en Estados Unidos

Otra característica importante que se debe resaltar es la relacionada con el tiempo de estancia de los mexicanos en Estados Unidos. El 59 por ciento de los migrantes mexicanos llevan más de diez años viviendo en Estados Unidos. Una pequeña proporción, registra una resiente llegada (menos de un año) a su lugar de destino. Entre la población informada, el promedio de tiempo viviendo en Estados Unidos fue de 21 años (Figura 2). 
Figura 2: Proporción de la población informada por tiempo vivido en Estados Unidos. Unidades Móviles de Salud y Bienestar, marzo a agosto de 2020



Meses

Menos de 5 años

- 6 - 10 años

- 11 - 15 años

- 16 - 20 años

Mayor de 20 años

- Sin información

Fuente: elaboración propia a partir de información en Base de Datos UMSB 2020.

\section{Nivel educativo}

En cuanto al nivel educativo, 55 por ciento tiene de uno a 12 años de escuela completa y más de la mitad de la población terminó al menos la preparatoria. Así mismo, una quinta parte alcanzó un nivel educativo universitario o de posgrado y uno por ciento no asistió a la escuela (Figura 3).

Nivel de inglés

El manejo del idioma puede ser un indicador de la forma como se inserta el migrante en la comunidad de destino. A nivel general se destaca que una cuarta parte de la población tiene un buen dominio del inglés y más de la tercera parte tiene un nivel básico de dicho idioma (Figura 4).

\section{Fuerza laboral}

En cuanto a la ocupación de las personas incluídas en la fuerza laboral se destaca que la mayoría desempeña labores en empleos de bajo nivel de exigencia 20 por ciento se ocupa en el áreas como la construcción, limpieza, agricultura, cocina o fábricas. Al indagar sobre el ingreso mensual promedio, 26 por ciento refirió recibir al menos mil dólares al mes y 60 por ciento menos de tres mil dólares mensuales (Figura 5). 
Figura 3: Proporción de la población informada según nivel educativo. Unidades Móviles de Salud y Bienestar, marzo a agosto de 2020

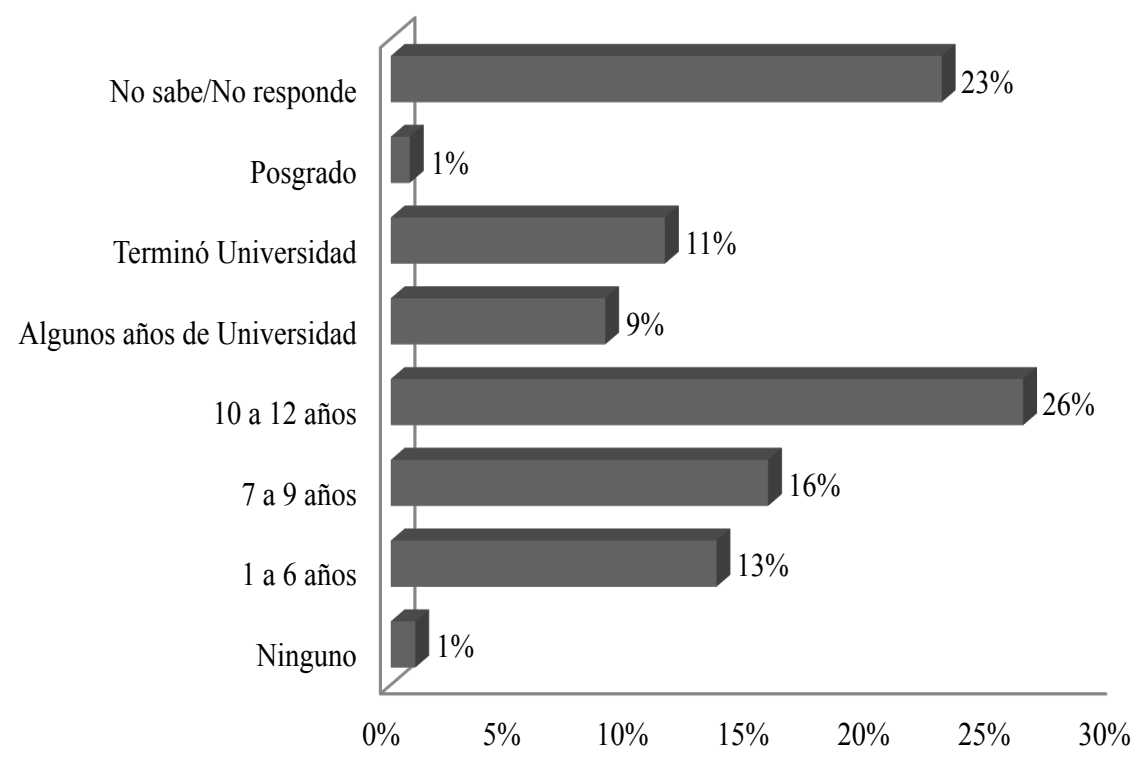

Fuente: elaboración propia a partir de información en Base de Datos UMSB 2020.

Figura 4: Proporción de la población informada según nivel de inglés. Unidades Móviles de Salud y Bienestar, marzo a agosto de 2020

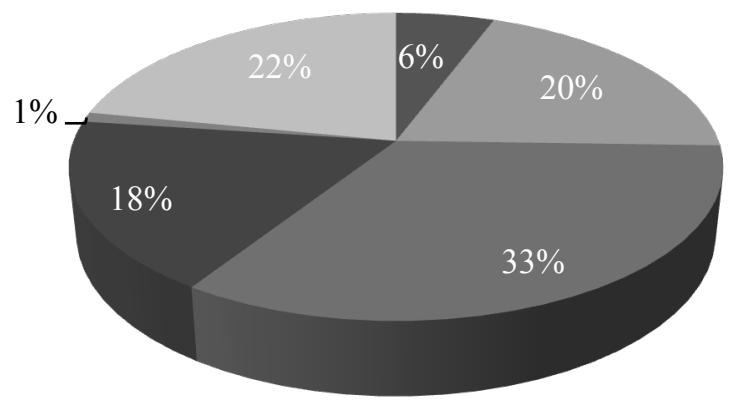
Muy bien
Bien
No bien
- Para nada
No sabe
Rehusó

Fuente: elaboración propia a partir de información en Base de Datos UMSB 2020. 
Respuesta frente al Covid-19 para la atención de la población migrante mexicana ... / A.M. LÓPEZ JARAMILLO et al.

Figura 5: Proporción de la población informada según ingresos mensuales.

Unidades Móviles de Salud y Bienestar, marzo a agosto de 2020

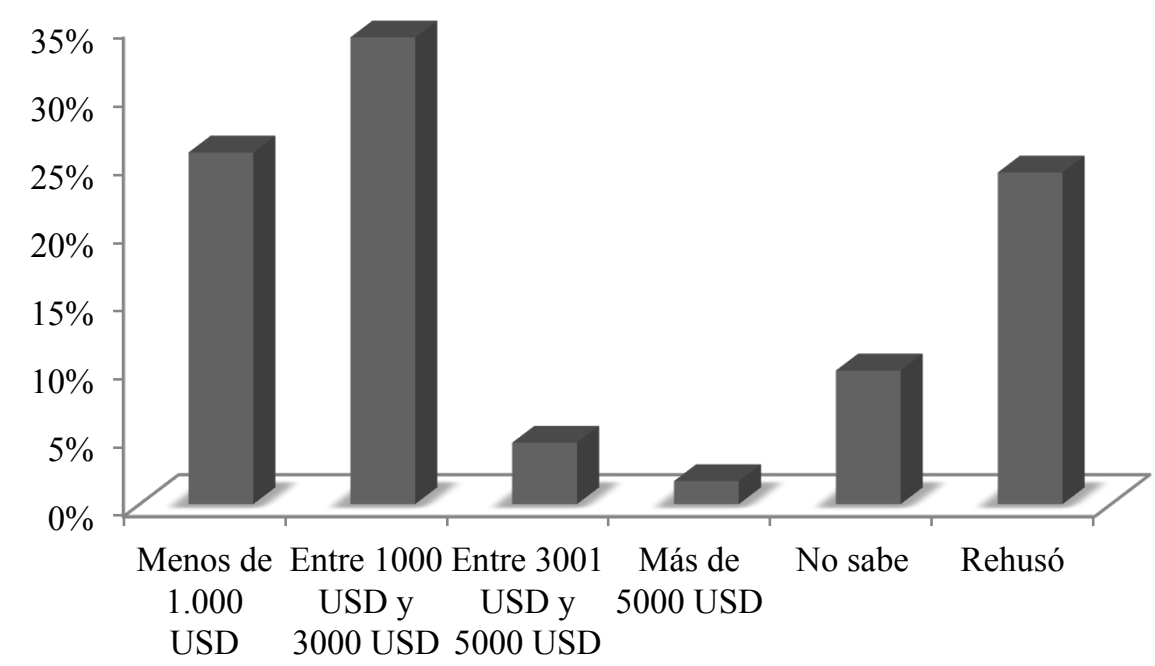

Fuente: elaboración propia a partir de información en Base de Datos UMSB 2020.

\section{Antecedentes personales}

A partir de la información de la base de datos de registro de las atenciones, también se pudieron conocer algunos antecedentes personales. Entre los cuales destaca que más de la mitad tienen antecedentes familiares como la diabetes mellitus, más de 40 por ciento considera que su alimentación es moderada y casi una quinta parte, reconoce consumir alimentos con alto nivel de azúcar, grasas y harinas. Finalmente, casi 70 por ciento reconoce realizar escasa actividad física moderada o escasa (Tablas 3, 4 y 5).

Tabla 3: Antecedentes personales de la población informada, marzo a agosto de 2020

\begin{tabular}{lrr}
\hline Alguien en su familia padece o ha padecido & Frecuencia & $\%$ \\
\hline Diabetes mellitus (Azúcar en la sangre) & 488 & 55 \\
Obesidad & 120 & 13 \\
Cáncer (próstata, mama, cuello cérvico-uterino) & 253 & 28 \\
No sé/No responde & 30 & 3 \\
Total & 891 & 100 \\
\hline
\end{tabular}

Fuente: elaboración propia a partir de información en Base de Datos UMSB 2020. 
Tabla 4: Autopercepción en la alimentación de la población informada, marzo a agosto de 2020

\begin{tabular}{lrr}
\hline Usted, ¿cómo considera su alimentación? & Frecuencia & $\%$ \\
\hline Balanceada & 224 & 25 \\
Moderada & 366 & 41 \\
Alto nivel en el consumo de azúcar, grasas y harinas & 185 & 21 \\
No responde & 116 & 13 \\
Total & 891 & 100 \\
\hline
\end{tabular}

Fuente: elaboración propia a partir de información en Base de Datos UMSB 2020

Tabla 5: Autopercepción en realización de actividad física de la población informada, marzo a agosto de 2020

\begin{tabular}{lrr}
\hline Usted, ¿cómo considera su actividad física? & Frecuencia & $\%$ \\
\hline Suficiente & 172 & 19 \\
Moderada & 311 & 35 \\
Escasa & 294 & 33 \\
No responde & 114 & 13 \\
Total & 891 & 100 \\
\hline
\end{tabular}

Fuente: elaboración propia a partir de información en Base de Datos UMSB 2020

En cuanto a comportamientos riesgosos, las personas informadas resaltaron que al menos 57 por ciento tuvieron relaciones sexuales sin protección, y alrededor de 80 por ciento manifestó no consumir tabaco o alguna sustancia psicoactiva (Tabla 6).

Tabla 6: Autopercepción en realización de actividad física de la población informada, marzo a agosto de 2020

\begin{tabular}{|c|c|c|c|c|c|c|c|}
\hline \multirow[t]{2}{*}{ Variable } & \multicolumn{2}{|c|}{$\mathrm{Si}$} & \multicolumn{2}{|c|}{ No } & \multicolumn{2}{|c|}{$\begin{array}{l}\text { No Sabe/No } \\
\text { responde }\end{array}$} & \multirow[t]{2}{*}{ Total } \\
\hline & Frec & $\%$ & Frec & $\%$ & Frec & $\%$ & \\
\hline $\begin{array}{l}\text { Usted tiene o ha tenido } \\
\text { relaciones sexuales sin } \\
\text { protección? }\end{array}$ & 507 & $5 \%$ & 255 & 29 & 129 & 14 & 891 \\
\hline Usted, ¿consume tabaco? & 44 & 5 & 727 & 82 & 120 & 13 & 891 \\
\hline $\begin{array}{l}\text { Usted consume alguna } \\
\text { sustancia psicoactiva? }\end{array}$ & 8 & 1 & 757 & 85 & 126 & 14 & 891 \\
\hline
\end{tabular}

Fuente: elaboración propia a partir de información en Base de Datos UMSB 2020 
Respuesta frente al Covid-19 para la atención de la población migrante mexicana ... / A.M. LÓPEZ JARAMILLO et al.

\section{Información relacionada con Covid-19}

En cuanto a la información relacionado con el Covid-19, se desataca que la totalidad de las personas a las que se les proporcionó información en las dos UMSB, han oído hablar del coronavirus o Covid-19. Así mismo, destaca que la mayoría de las personas informadas consideran que su nivel de información es bueno o muy bueno, su principal fuente de informació es la televisión ( 58 por ciento) y más de 70 por ciento consideran que la situación es grave (Tablas 7, 8 y 9).

Tabla 7: Autopercepción del nivel de información de la población informada, marzo a agosto de 2020

\begin{tabular}{lrr}
\hline $\begin{array}{l}\text { ¿Cómo consideraría su nivel de } \\
\text { información sobre qué es Covid-19? }\end{array}$ & Frecuencia & $\%$ \\
\hline Muy bueno & 285 & 60 \\
Bueno & 153 & 32 \\
Regular & 32 & 7 \\
No informado & 3 & 1 \\
Total & 473 & 100 \\
\hline
\end{tabular}

Fuente: elaboración propia a partir de información en Base de Datos UMSB 2020.

Tabla 8: Fuente de información sobre Covid-19 de la población informada, marzo a agosto de 2020

\begin{tabular}{lrr}
\hline ¿Cuál es su principal fuente de & Frecuencia & $\%$ \\
información sobre Covid-19? & 7 & 1 \\
\hline Radio & 272 & 58 \\
Televisión & 68 & 14 \\
Redes sociales & 103 & 22 \\
Familia/amigos/vecinos & 20 & 4 \\
Otro & 470 & 100 \\
Total & 470 \\
\hline
\end{tabular}

Fuente: elaboración propia a partir de información en Base de Datos UMSB 2020. 
Tabla 9: Autopercepción de gravedad del Covid-19 de la población informada, marzo a agosto de 2020

\begin{tabular}{lrr}
\hline ¿Qué tan grave cree que es la situación actual? & Frecuencia & $\%$ \\
\hline Muy grave & 338 & 73 \\
Grave & 117 & 25 \\
No es grave & 0 & 0 \\
No creo que exista & 7 & 2 \\
Total & 462 & 100 \\
\hline
\end{tabular}

Fuente: elaboración propia a partir de información en Base de Datos UMSB 2020.

En cuanto a la relación del brote del Covid-19 y el empleo, casi una quinta parte (18 por ciento) de las personas informadas perdieron su trabajo durante el periodo del reporte y en igual proporción, algún familiar también perdió el empleo (Tablas 10 y 11).

Tabla 10: Pérdida del trabajo debido al brote del Covid-19 de la población informada, marzo a agosto de 2020

\begin{tabular}{lrr}
\hline ¿Perdió su trabajo debido al brote de Covid-19? & Frecuencia & $\%$ \\
\hline $\mathrm{Si}$ & 85 & 18 \\
$\mathrm{No}$ & 375 & 82 \\
Total & 460 & 100 \\
\hline
\end{tabular}

Fuente: elaboración propia a partir de información en Base de Datos UMSB 2020.

Tabla 11: Pérdida del trabajo del agún familiar debido al brote del Covid-19 de la población informada, marzo a agosto de 2020

\begin{tabular}{lrr}
\hline $\begin{array}{l}\text { ¿Algún miembro de su familia perdió su trabajo } \\
\text { debido al brote de Covid-19? }\end{array}$ & Frecuencia & $\%$ \\
\hline $\mathrm{Si}$ & 85 & 18 \\
No & 375 & 82 \\
Total & 460 & 100 \\
\hline
\end{tabular}

Fuente: elaboración propia a partir de información en Base de Datos UMSB 2020.

Con respecto a las medidas de protección implementadas por las personas informadas, resalta que entre marzo y agosto de 2020, casi en su totalidad ( 99.5 por ciento) se quedan en casa el mayor de tiempo posible, mantiene una distancia adecuada respecto a las demás personas (98 por ciento), utiliza las medidas de higiene sugeridas (lavado de manos o uso de 
Respuesta frente al Covid-19 para la atención de la población migrante mexicana ... / A.M. LÓPEZ JARAMILLO et al.

gel antibacterial), evitan el contacto de ojos, nariz y boca, y usan la mascarilla cuando están fuera de casa (Tabla 12).

Tabla 12: Medidas de protección implementadas debido al brote del Covid-19 de la población informada, marzo a agosto de 2020

\begin{tabular}{lrr}
\hline Ustedes y las personas que viven en su hogar & Frecuencia & $\%$ \\
\hline Se queda la mayor parte del tiempo dentro de su casa & 458 & 99.5 \\
Mantiene seis pies de distancia con otras personas & 452 & 98 \\
fuera de casa & 424 & 92 \\
Se lavan las manos por 20 segundos & 442 & 96 \\
Evita el contacto con otros si es posible & 410 & 89 \\
Usa mascarilla cuando se encuentra fuera de casa & 403 & 88 \\
Usa cloro/alcohol para limpiar las áreas comunes en & & \\
su casa & 380 & 83 \\
Usa gel sanitizante de 60\% de alcohol o mayor para & 387 & 84 \\
desinfectar sus manos & 460 & \\
Evita tocar sus ojos, boca y nariz & & \\
Total & & \\
\hline
\end{tabular}

Fuente: elaboración propia a partir de información en Base de Datos UMSB 2020.

Finalmente, al indagar sobre el impacto del brote del Covid-19 sobre la salud mental de las personas informadas en las UMSB, destaca que más de la mitad se sentía más aislado durante este periodo ( 58 por ciento), una cuarta parte se sentía más cansado ( 26 por ciento), casi una quinta parte más estresado (19 por ciento) y en menores proporciones reportaron sentirse solos, tristes, ansiosos o preocupados por el futuro (Tabla 13).

Los anteriores resultados se refuerzan con los hallazgos de estudios realizados durante el presente año, en los cuales destacan que es posible que los migrantes tengan menos probabilidades de hacerse la prueba de Covid-19, y que quienes se someten a la prueba tienen probabilidades de estar enfermos $y$, por lo tanto, tienen indicaciones clínicas para realizar la prueba. Así mismo, resaltan que en la población latina son los factores estructurales como ser hispanohablantes monolingües, el desempleo, las enfermedades cardiacas y un menor distanciamiento social, los que se asocian con un mayor riesgo de contraer el Covid-19, y en cuanto al riesgo a morir por dicha enfermedad, destacan características como un mayor número de integrantes del hogar, la contaminación del aire, el desempleo y ser menor 
de 35 años (Rodriguez-Diaz CE et al., 2020). Así mismo, la CDC (2020) resalta que padecimientos como la obesidad, diabetes mellitus, hipertensión, enfermedad pulmonar y enfermedad cardiovascular están presentes entre poblaciones afroestadounidenses y latinas, preexistencias que pueden convertir a estas poblaciones en altamente vulnerables a este virus.

Tabla 13: Autopercepción de la salud mental de la población informada debido al brote del Covid-19, marzo a agosto de 2020

\begin{tabular}{lrr}
\hline $\begin{array}{l}\text { Comparando cómo se siente hoy con la forma en } \\
\text { que se sentía en febrero, usted diría que está }\end{array}$ & Frecuencia & $\%$ \\
\hline Más estresado & 88 & 19 \\
Más ansioso & 44 & 10 \\
Más triste & 27 & 6 \\
Más solo & 26 & 6 \\
Más aislado & 273 & 59 \\
Más cansado & 119 & 26 \\
Más preocupado por el futuro & 35 & 8 \\
Total & 460 & \\
\hline
\end{tabular}

Fuente: elaboración propia a partir de información en Base de Datos UMSB 2020

Según Garg et al. (2020), entre los afroestadounidenses y latinos, la expansión del Covid-19 se debe principalmente a la proximidad física que mantienen dadas las condiciones de hacinamiento en las que viven. Así mismo, los bajos ingresos les exigen asistir a sus trabajos, lo cual puede incrementar el riesgo de contagio.

Por otra parte, en un estudio realizado entre familias latinas de agricultores y no agricultores, se encontró que el conocimiento de Covid-19 y los métodos de prevención fue alto en ambos grupos, al igual que la percepción de su gravedad. Ambos grupos afirmaron practicar comportamientos preventivos, aunque las familias de agricultores enfatizaron en el distanciamiento social y las familias de los no agricultores enfatizaron en la higiene personal. Las interacciones sociales detalladas mostraron altas tasas de contacto interpersonal en el hogar, en el trabajo y en la comunidad, con más uso de máscaras en personas que no son familias de agricultores. Finalmente, a pesar de los altos niveles de conocimiento y la gravedad percibida del Covid-19, estas familias inmigrantes estaban involucradas en un contacto interpersonal frecuente que podría exponer a los miembros de la comunidad y a ellos mismos al Covid-19 (Quandt SA et al., 2020). 
Otros autores se han centrado en resaltar las desigualdades y disparidades en salud entre las minorías y destacan que comunidades negras e hispanas están expuestas al Covid-19 con mayor frecuencia como resultado del racismo estructural. Así mismo, resaltan que las minorías realizan trabajos más riesgosos y en condiciones peligrosas, como trabajar en la "primera línea" de combate contra el coronavirus, es decir, en áreas como la alimentación, transporte, farmacias o limpieza. Debido a esto, están entrando en contacto con Covid-19 antes que otros grupos raciales y experimentando sus impactos antes en la pandemia (Cione et al., 2020; Caicedo, 2020; NAE, 2020; Navarro, 2020).

\section{RETOS HACIA EL FUTURO Y RECOMENDACIONES}

Entre los retos que se han identificado, destaca la continuidad de los servicios preventivos de salud, así como las referencias, haciendo especial énfasis a las comunidades vulnerables, respetando las medidas de seguridad, tales como el distanciamiento social, lo cual debe considerar la disminución de eventos comunitarios, tales como ferias de salud o conferencias, entre otros. Para ello, es importante integrar el trabajo remoto y apoyarse de herramientas tecnológicas que permitan la comunicación a distancia. Y poner especial atención en la corrección de información falsa.

Así mismo se presenta el reto de realizar seguimiento de casos sospechosos y positivos, para brindar atención oportuna y disminuir la propagación.

Destaca la necesidad de fortalecer el cuidado al personal de salud, mediante la suficiencia de insumos de protección y equipo, así como aumentar el personal sanitario en el segundo nivel de atención.

A este respecto, otro reto importante considera la atención a la salud mental que ha tomado gran relevancia ante la actual contingencia.

Específicamente en la frontera México-Estados Unidos, es importante fortalecer la capacitación para agilizar el manejo e intercambio de información de casos binacionales, así como identificar el momento adecuado para la reactivación social y económica, lo cual tendrá un impacto directo económico y social.

Ante este panorama, surgen diversas recomendaciones para tomar acción en beneficio de la población, entre ellas destaca actualizar el directorio de aliados estratégicos, y mantener y fortalecer la vinculación con aliados locales de salud, para contribuir en la ampliación de referencias (atención médica por Covid-19 u otros padecimientos crónicos), así como fortalecer el acceso a la atención médica, con especial énfasis en la población mi- 
grante. Identificar los recursos adicionales para la comunidad migrante, tales como ayudas de alimentos, apoyos económicos, casos de violencia doméstica, asesoría laboral, entre otros; y fortalecer las alianzas en temas de salud mental para favorecer modelos de atención comunitaria, grupos de apoyo y referencias a consejería. Reforzar la atención a la población migrante en situación de vulnerabilidad

Otro punto importante es aumentar la comunicación, coordinación, y colaboración dentro de México, así como con Estados Unidos, para avanzar juntos en la lucha contra la Covid-19 y fortalecer la atención a las poblaciones móviles. Asimismo, mantener la vigilancia y seguimiento de casos sospechosos y positivos, para brindar atención oportuna, así como fortalecer la vigilancia y seguimiento de la Covid-19 en poblaciones vulnerables como personas con diabetes, obesidad, hipertensión, migrantes y pueblos originarios.

Implementar un programa de reactivación social y económico de manera gradual, ordenada y cauta, para evitar el aumento de casos.

Como siguientes pasos se identifica la preparación para brindar servicios preventivos de manera virtual; fortalecer las capacidades para brindar servicios de orientación, referencia y seguimiento; fortalecer el tamizaje de salud mental; así como fortalecer la capacidad técnica del personal de las UMSB en diversos proyectos estratégicos, entre los que se incluye la salud mental.

\section{Conclusiones}

A partir de la contigencia de salud a nivel mundial, los retos que ésta implica, y teniendo en cuenta la importancia de conocer y mejorar la salud de las y los migrantes mexicanos en Estados Unidos, las Unidades Móviles de Salud y Bienestar iniciaron una prueba piloto en las UMSB de Tucson y Phoenix, para adaptar los servicios en materia de Covid-19, los cualen incluyen orientación, detección y referencia. Así, el principal objetivo del presente artículo fue describir la respuesta y los retos binacionales frente al Covid-19 y presentar el perfil demográfico de los migrantes en Estados Unidos que recibieron información sobre Covid-19 entre marzo y agosto de 2020 en las UMSB.

En cuanto a la respuesta, los actores gubernamentales de la región fronteriza México-Estados Unidos, implementaron diversas acciones y buenas prácticas en materia de Covid-19, entre las que destaca el fortalecimiento de la capacidad técnica del personal de salud y otros involucrados, acciones específicas a distancia de apoyo a la salud mental durante la pandemia, 
el desarrollo de material educativo de prevención, la implementación de filtros ambulatorios, clínicas y hospitales temporales, así como la ejecución de clínicas de fiebre en el primer nivel de atención, entre otros.

Se mantiene una vinculación y articulación interinstitucional en los estados mexicanos para el monitoreo y atención médica a la población migrante en estaciones migratorias, repatriación y albergues. A nivel binacional, destaca la activación del Protocolo Operacional para la Comunicación y Coordinación Binacional entre México y Estados Unidos sobre Notificaciones de Enfermedades y Brotes, además de la coordinación y comunicación entre estados de ambos lados de la frontera para el intercambio de información diaria y el manejo de casos binacionales.

Referente al perfil sociodemográfico de los migrantes mexicanos que recibieron información sobre el Covid-19 en las UMSB de Tucson y Phoenix entre marzo y agosto de 2020 es principalmente, población femenina entre 25 y 49 años, es decir, en edad laboralmente activa, procedente del estado fronterizo de Sonora, con un promedio de estancia en Estados Unidos de 12 años, un nivel educativo menor a la preparatoria y un bajo nivel de inglés. En cuanto a la situación laboral, se ocupan en oficios de baja remuneración. Estos hallazgos se refuerzan con los estudios realizados por diversos autores, los cuales destacan por ejemplo, ser latinos monolingües, ser menores de 35 años, ser agricultores o tener bajos ingresos como algunos factores de riesgo para contraer Covid-19 (Rodriguez-Diaz et al., 2020; Garg et al., 2020; Quandt SA et al., 2020).

Entre los antecedentes personales se destaca que más de la mitad de la población reportó tener diabetes mellitus, una alimentación moderada o alta en grasas y azúcares y con poca actividad física como lo destaca el estudio de CDC (2020).

Específicamente, con relación al Covid-19, se resalta que la población se condisera bien informada, que la situación es grave y que son necesarias las medidas de protección y distanciamiento social para evitar contraer el virus como lo demuestra el estudio de Quandt SA et al. (2020).

Finalmente, entre los retos se considera la atención a la población migrante en situación de vulnerabilidad, así como dar continuidad de los servicios preventivos de salud y referencias, respetando las medidas de seguridad, tales como el distanciamiento social. Para lo cual, se vuelve un reto integrar a la forma de trabajo herramientas digitales que permitan la comunicación a distancia, el seguimiento de casos sospechosos y positivos, así como fortalecer la atención a la salud mental que ha tomado gran relevancia ante la actual contingencia. Referente al trabajo específico en 
la frontera México-Estados Unidos, destaca la necesidad de fortalecer el intercambio de información de casos binacionales, así como identificar el momento adecuado para la reactivación social y económica, lo cual tendrá un impacto directo económico y social.

\section{REFERENCIAS BIBLIOGRÁFICAS}

Caicedo, Maritza, 2020, "Covid-19 y desigualdad social en Estados Unidos", en Este país. Revista digital, 27 de mayo de 2020, Fecha de consulta: 20/08/2020. Disponible en https://estepais.com/tendencias_y_opiniones/covid-19-y-desigualdad-social-en-estados-unidos/

Centers for Disease Control and Prevention (CDC), 2020, National Diabetes Statistics Report, 2020. Atlanta, GA: Centers for Disease Control and Prevention, US Department of Health and Human Services. Disponible en https://www.cdc.gov/ diabetes/pdfs/data/statistics/national-diabetes-statistics-report.pdf

Centers for Disease Control and Prevention (CDC), 2020, United States COVID-19 Cases and Deaths by State, 27 de octubre de 2020, Atlanta, GA: Centers for Disease Control and Prevention, US Department of Health and Human Services. Disponible en https://covid.cdc.gov/covid-data-tracker/index.html\#cases_casesper100k

Cione, C., Castaneda, E., Ferdinando, A., Prince, J., Jackson, D., Vetter, E., McCarthy, S., 2020, Covid-19 Susceptibility Among Latin People in El Paso, TX. SSRN, Jun:3608396. Disponible en DOI: 10.2139/ssrn.3608396.

Dirección General de Epidemiología, 2020, Datos Covid-19 en México. Disponible en https://datos.covid-19.conacyt.mx/ (Fecha de consulta: 27/10/2020)

Garg S, Kim L, Whitaker M. et al., 2020, Hospitalization Rates and Characteristics of Patients Hospitalized with Laboratory-Confirmed Coronavirus Disease 2019 - Covid-NET, 14 States, March 1-30, 2020. MMWR Morb Mortal Wkly Rep 2020;69:458-464. Disponible en http://dx.doi.org/10.15585/mmwr. mm6915e3

Quandt, S.A.; LaMonto, N.J.; Mora, D.C.; Talton, J.W.; Laurienti, P.J.; Arcury, T.A., 2020, "Covid-19 Pandemic among Latinx Farmworker and Nonfarmworker Families in North Carolina: Knowledge, Risk Perceptions, and Preventive Behaviors", in Int. J. Environ. Res. Public Health, 17(16): 5786. Disponible en doi: $10.3390 /$ ijerph 17165786

New American Economy, 2020, Undocumented Immigrants and the Covid-19 Crisis. Research Fund, April 4. Disponible en https://research.newamericaneconomy.org/report/undocumented-immigrants-covid-19-crisis/

Navarro, M., 2020, "Migrantes mexicanos en EU: solos ante Covid-19, crisis y políticas antiinmigrantes", en Forbes México, Revista digital, 29 de abril de 2020. Fecha de consulta: 20/08/2020. Disponible en https://www.forbes.com.mx/noticias-migrantes-mexicanos-eu-desamparados-coronavirus/ 
Respuesta frente al Covid-19 para la atención de la población migrante mexicana ... / A.M. LÓPEZ JARAMILLO et al.

Rodriguez-Diaz, CE. et al., 2020, "Risk for Covid-19 infection and death among Latinos in the United States: examining heterogeneity in transmission dynamics", in Annals of Epidemiology. S1047-2797(20)30267-2. Disponible en https://doi. org/10.1016/j.annepidem.2020.07.007

Secretaría de Relaciones Exteriores, 2020, Actualización sobre el seguimiento de connacionales con Covid-19 en EE. UU. Disponible en https://www.gob.mx/sre/ documentos/nota-informativa-relaciones-exteriores-no-36 (Fecha de consulta: $31 / 08 / 2020$ ).

\section{RESUMEN CURRICULAR DE LOS AUTORES}

\section{Ana María López Jaramillo}

Doctora en Ciencias Sociales con especialidad en Estudios Regionales por El Colegio de la Frontera Norte, institución en la que está a cargo de la Coordinación Académica del Doctorado en Ciencias Sociales en el Área de Estudios Regionales. Recientemente, desarrolló proyectos de investigación relacionados con Migración y Salud en la Comisión de Salud Fronteriza México - Estados Unidos y realizó el proyecto de investigación Building Binational Partnerships to Address Rural Mexican Migrants' Healthcare Service Needs con fondos del Programa de Investigación y Salud (PIMSA) 2017-2018 en asocio con la Universidad de California, Riverside. Pertenece a la Sociedad Mexicana de Demografía (SOMEDE), a la Asociación Latinoamericana de Población (ALAP), a la Red Latinoamericana de Estudios Fronterizos (RELATEF) y a la Red Migración y Salud de Colombia. Dirección electrónica: alopez@ colef.mx

Registro ORCID: https://orcid.org/0000-0003-3342-2982

\section{María Gudelia Rangel Gómez}

Profesora investigadora de El Colegio de la Frontera Norte. Es Doctora en Ciencias de la Salud por el Instituto Nacional de Salud Pública, con especialidad en Epidemiología, Maestra en Salud Pública por la Universidad Autónoma de Baja California y Licenciada en Trabajo Social. Dentro de su trayectoria académica ha dirigido tesis de investigación a nivel de maestría y doctorado en temas de salud pública y epidemiología, tales como: VIH/ SIDA, salud reproductiva, acceso a servicios de salud, abuso de sustancias, obesidad, salud y migración y salud fronteriza. Igualmente, colabora como investigadora responsable en varios proyectos de investigación binacional en temas de salud pública y epidemiología, ha publicado múltiples investigaciones en revistas especializadas a nivel internacional y capítulos de libros. Es investigadora nivel I en el Sistema Nacional de Investigadores 
(SNI); y es miembro del Comité Directivo de la Iniciativa Conjunta de Salud y Migración para Centroamérica y México (INCOSAMI). También es miembro del Consejo Directivo del Programa de Investigación en Salud y Migración (PIMSA) de la Universidad de California.

Dirección electrónica: grangel@colef.mx

Registro ORCID: https://orcid.org/0000-0003-3376-152X

\section{Rodolfo Cruz Piñeiro}

Doctorado en Sociología con especialidad en Población por la Universidad de Texas en Austin; Maestría en Demografía por El Colegio de México; miembro del Sistema Nacional de Investigadores, Nivel III. Director del Departamento de Estudios de Población y Director de la revista Migraciones Internacionales de El Colegio de la Frontera Norte. Fue presidente de la Sociedad Mexicana de Demografía (SOMEDE) 2002-2004. Sus principales áreas de estudio son: Migración interna e internacional, mercados laborales; población y desarrollo en la frontera de México y los Estados Unidos. Cuenta con la publicación de un libro, cinco coordinaciones de libros y más de 100 capítulos y artículos en revistas especializadas. La mayor parte de sus estudios y publicaciones centran su atención en el fenómeno de la migración interna e internacional. Ha impartido cursos de análisis demográfico, teoría social de la población, metodología de la migración y fuerza laboral, entre otros. Asimismo, ha sido profesor visitante en la Universidad de Texas en Austin y en la Universidad Estatal de San Diego. Dirección electrónica: rcruz@colef.mx Registro ORCID: https://orcid.org/0000-0001-6770-0413

Artículo recibido el 20 de noviembre de 2020 y aprobado el 16 de febrero de 2021 . 